\title{
Verbesserung der Ephemeride von Y Cygni.
}

Sind die von Prof. Dunér (A. N. 3720) angegebenen Zeiten auf Greenwich bezogen, wie man wohl vermuthen darf, so weichen meine Bestimmungen nicht unerheblich von den in Upsala gemachten ab. Ich erhielt folgende Reihe von Minimis gerader Zählung:

\begin{tabular}{|c|c|c|c|}
\hline & & $1900-01$ & M.Z. Greenw \\
\hline Epoche & 3402 & Nov. 23 & $8^{\mathrm{h}} 29^{\mathrm{m}}$ \\
\hline$»$ & 3432 & Jan. 7 & 626 \\
\hline$\triangleright$ & 3434 & 10 & $63^{6}$ \\
\hline$»$ & 3436 & 13 & 71 \\
\hline$»$ & $343^{8}$ & 16 & 646 \\
\hline
\end{tabular}

Aus dieser nicht unter sehr günstigen atmosphärischen Verhältnissen gewonnenen Reihe folgt als mittlere Correction der Hartwig'schen Ephemeride $+36^{\mathrm{m}} \cdot 2$; und da die Ephemeride etwa um $8^{\mathrm{m}} .7$ kleinere Zeiten angiebt als Dunér's Ephemeride in

Münster, I 90 I Juni 13 . dem (mir nicht zugänglichen) Astroph. Journal, so ist letztere noch immer um $+27^{\mathrm{m}}$ bis $+28^{\mathrm{m}}$ zu verbessern. In Verbindung mit der aus Duner's Reihe hervorgehenden Thatsache, dass er die Minima um $13^{\mathrm{m}}$ eher eintreten sieht als Bergstrand, ist das ein merkwürdiger Beweis für die Verschiedenheit der Auffassungen. - Das ungerade Minimum Epoche 35 I 7 beobachtete ich 1901 Mai $149^{\mathrm{h}} 59^{\mathrm{m}}$ Greenw., Correction der Hartwig'schen Ephemeride $+30^{\mathrm{m}}$, etwas unsicher, weil erst von $9^{\mathrm{h}} 17^{\mathrm{m}}$ an beobachtet werden konnte. Dagegen trat das Minimum Epoche 3437, 1901 Jan. I 4 mit einer mehrstündigen Verfrühung ein, die zwar durch Wolken erklärt werden könnte, sich jedoch Januar 17 noch stärker ausgeprägt zeigte. Vielleicht ist in beiden Fällen nicht das wahre Minimum, sondern ein vorhergehendes Theilminimum beobachtet worden.

\section{Correction to Klinkerfues' Theoretische Astronomie.}

In the hundertundachte Vorlesung of Klinkerfues' Theoretische Astronomie there is given for determining the node of a double star orbit the equation (5)

$$
\sec 2 \zeta=\operatorname{cotang}(B-A) \operatorname{tang}(B+A-2 \Omega)
$$

and the same equation is reproduced, with altered sign and some slight change of form, in See's Evolution of the Stellar Systems I. But in neither of these works is the equation

Washburn Observatory, I go I June. correctly given as I have found by attempting to apply it to numerical computation and the error appears to have been produced by neglecting a minus sign in Klinkerfues' Equation 4 from which this is derived. The correct form, which should be substituted in both of the above named works, is

$$
\cos 2 \zeta=\operatorname{cotang}(A-B) \operatorname{tang}(A+B-2 \Omega) \text {. }
$$

George C. Comstock.

Zusatz des Herausgebers. Die berichtigte Form ist mir schon seit längerer Zeit bekannt, wahrscheinlich wird sie auch anderen Astronomen nicht entgangen sein. In der II. Auflage von Klinkerfues Theoretische Astronomie ist die Gleichung (5) bis auf das Vorzeichen der rechten Seite verbessert wiedergegeben.

\section{Endeckung von fünf neuen Planeten.}

1901 GV. Planet Villiger I 1. October $10^{\mathrm{h}} 26^{\mathrm{m}} 6 \mathrm{M}$. Z. München RA. $=29^{\circ} 36^{\circ}$ PD. $=90^{\circ} 30^{\circ}$ tägl. Bewegung $-12^{\prime}+9^{\prime}$ Gr. $11^{m} \cdot 5$. Villiger.

1901 GX. Planet Carnera-Kopff I r. October $13^{\text {h }} \mathbf{I I}^{\mathbf{m}} \cdot 7$ M. Z. Heidelberg RA. $=35^{\circ} 11^{\prime} \mathrm{PD}=93^{\circ} 10^{\prime}$ tägl. Bewegung $-12^{\prime}+9^{\prime}$ Gr. $13^{\mathrm{m}}$. Carnera.

rgor GY. Planet Carnera-Kopff 11 . October $10^{\mathrm{h}} 19^{\mathrm{m}} \cdot 5 \mathrm{M}$. Z. Heidelberg RA. $=35^{\circ} 39^{\circ} \mathrm{PD}=91^{\circ} 11^{\prime}$ tägl. Bewegung $-\mathrm{I2}^{\prime}+5^{\prime}$ Gr. $122^{\mathrm{m}} 8$. Carnera.

1901 GZ. Planet Kopff 13. October $13^{\mathrm{h}} 0^{\mathrm{m}} 9 \mathrm{M}$. Z. Heidelberg RA. $=27^{\circ} \mathbf{6}^{\prime}$ PD. $=71^{\circ} 51^{\prime}$ tägl. Bewegung $-16^{\prime}+2^{\prime}$ Gr. 12 $2^{\mathrm{m}} 5$. Carnera.

I 901 HA. Planet Carnera.Kopff 13 . October $1_{1^{\mathrm{h}}}^{\mathrm{h}} 39^{\mathrm{m}} \cdot 5 \mathrm{M} . \mathrm{Z}$. Heidelberg RA. $=353^{\circ} \circ^{\circ} \mathrm{PD} .=95^{\circ} 15^{\circ}$ tägl. Bewegung $-9^{\prime}+4^{\prime}$ Gr. $13^{\mathrm{m}} \circ$. Carnera.

Ein der Centralstelle gemeldeter neuer Planet Carnera-Kopff 1901 GW ist identisch mit (247) Eukrate. Position: Oct. II I $3^{\mathrm{h}} \mathrm{I}^{\mathrm{m}}{ }^{\mathrm{m}} 7 \mathrm{M}$. Z. Heidelberg RA. $=353^{\circ} 5 \mathrm{I}^{\circ}$ PD. $=88^{\circ} 2 \mathrm{I}^{\circ}$ Gr. $\mathrm{II}^{\mathrm{m}}$.

Kiel, 1901 Oct. 14:

H. Kreutz.

Inhalt zu Nr. 3744. E. Hartwig. Ortsbestimmungen und Mittheilungen zu neuen veränderlichen Stersen. 369. - E. $v$. Oppolzer. Ueber den Gleichgewichtszustand der Sonnenatmosphäre. 375. - E. C. Pickering. Opposition of (433) Eros in 1903. $377 .-$ C. 7 . Merfield. Observations of Comet 190I I. 379. - L. F. Gruey. Observations de Ia planete I901 GQ. 381. - L. Ambronn. Nova (3.1901) Persei. 38r. - F. Plassmann. Vebesserung der Ephemeride von Y Cygni. 383. - G. C. Comstock. Correction to Klinkerfues' Theoretische Astronomie. 383. - Zusatz hierzu. 383. - II. Kreutz. Entdeckung von fúuf neuen Planeten. 383. Geschlossen rgor Oct. 15. Herausgeber: H. Kroutz. Druck von C. Schaidt. Expedition: Kiel, Nienannoweg ro3. 